\title{
Laparoscopy-Assisted Transperitoneal Percutaneous Nephrolithotomy for the Treatment of Renal Stones in a Horseshoe Kidney
}

This article was published in the following Dove Press journal: Research and Reports in Urology

\author{
Ramin Haghighi $\mathbb{D}^{\prime}$ \\ Abdolah Razi (iD) \\ Ashkan Haghighi ${ }^{2}$ \\ Navid Ebrahimipour (1D ${ }^{3}$ \\ Ali Teimouri $\mathbb{D}^{4}$ \\ 'Department of Urology, Faculty of \\ Medicine, North Khorasan University of \\ Medical Sciences, Bojnord, Iran; ${ }^{2}$ Student \\ Research Committee, North Khorasan \\ University of Medical Sciences, Bojnord, \\ Iran; ${ }^{3}$ Department of Emergency \\ Medicine, Faculty of Medicine, North \\ Khorasan University of Medical Sciences, \\ Bojnord, Iran; ${ }^{4}$ Department of \\ Emergency Medicine, Faculty of Medicine, \\ Mashhad University of Medical Sciences, \\ Mashhad, Iran
}

\begin{abstract}
The objective of this case report is to highlight treatment by LAT-PCNL in a patient with horseshoe kidney. A 51-year-man with hematuria presented to the emergency department with moderate abdominal pain starting $7 \mathrm{hrs}$ ago. He did not report a history of previous urological disease. Renal stone and horseshoe kidney malformation were diagnosed based on the plain abdominal film, intravenous urogram (IVU), non-contrast computed tomography (CT-IVU), and ultrasound findings. Due to the larger stone bulk and renal malformation, it was not possible to perform extracorporeal shock wave lithotripsy (ESWL) monotherapy or ESWL sandwich therapy. Moreover, since percutaneous nephrolithotomy (PCNL) had some challenges and limitations due to high skin-to-stone distance, special anatomy, dispersion stones, and possible consequent internal organs injuries, we performed LAT-PCNL on our patient. We did not observe any perioperative complication. Postoperative control abdominal x-ray revealed only a medium ureteral stone that was extracted while removing the Double-J stent a month later. The patient was discharged on the third postoperative day with normal laboratory values. At 3-month follow-up, the patient was stone-free with normal renal function and renal ultrasonography. Laparoscopy-assisted transperitoneal PCNL seems to be a safe and minimally invasive technique that can be used as an alternative approach in the management of renal stones in special cases of horseshoe kidneys.
\end{abstract}

Keywords: horseshoe kidney, laparoscopy-assisted, percutaneous nephrolithotomy

\section{Introduction}

Horseshoe kidney is one of the most common congenital renal anomalies of the genitourinary system with an incidence of 1 in 400 cases. ${ }^{1}$ The incidence of nephrolithiasis is reported between $21 \%$ and $60 \%$ in patients with horseshoe kidneys. ${ }^{2}$ The abnormal anatomical position of pelvis and calyces might lead to several complications. Although most of the affected patients are asymptomatic, they could be first manifested by infections, renal stones, or obstruction. Currently, percutaneous nephrolithotomy (PCNL) and somewhat extracorporeal shock wave lithotripsy (ESWL) are the common therapeutic methods recommended for horseshoe nephrolithiasis. PCNL is a preferred modality in the treatment of renal stones that are larger than $20 \mathrm{~mm}$ in diameter or the large stones failed to be treated by ESWL. However, the PCNL approach could pose a serious risk of injury to the aberrant vascular and adjacent organs, especially the overlying bowels. Thus, a more meticulous method such as laparoscopy could be of great help in this
Correspondence: Ali Teimouri Department of Emergency Medicine, Faculty of Medicine, Mashhad University of Medical Sciences, Mashhad, Iran Email ali.teimouri.1994@gmail.com 
procedure. Herein, we present a case of HSK with renal stones treated by Laparoscopy-assisted transperitoneal percutaneous nephrolithotomy (LAT-PCNL).

\section{Case Presentation}

A 51-year man presented to the Emergency Department with the chief complaint of dull and moderate abdominal pain starting $7 \mathrm{hrs}$ ago. He complained of concomitant nausea and hematuria. Results of all the laboratory tests including renal function tests were within normal limits except urinalysis that showed a urinary tract infection. A plain abdominal film was identified several renal stones on the right side of the lumbar vertebrae (Figure 1). Renal ultrasonography also revealed the stones accompanied by the severe dilatation of the right pelvicalyceal system. Afterward, intravenous urogram (IVU) and IVU with noncontrast computed tomography (CT-IVU) were performed, which confirmed the ultrasound findings and showed the renal anatomy (Figure 2). He did not have any previous urological diseases. Urinary tract infection was treated successfully.

Possible treatment modalities for HSK with large stones are PCNL, ESWL, laparoscopy, and occasionally open surgery. ${ }^{2}$ Due to the larger stone bulk and renal malformation, it was not possible to perform ESWL monotherapy or ESWL sandwich therapy. Moreover, PCNL has relative challenges and limitations because of high skin-to-stone distance, special anatomy, dispersion stones, and possible consequent internal

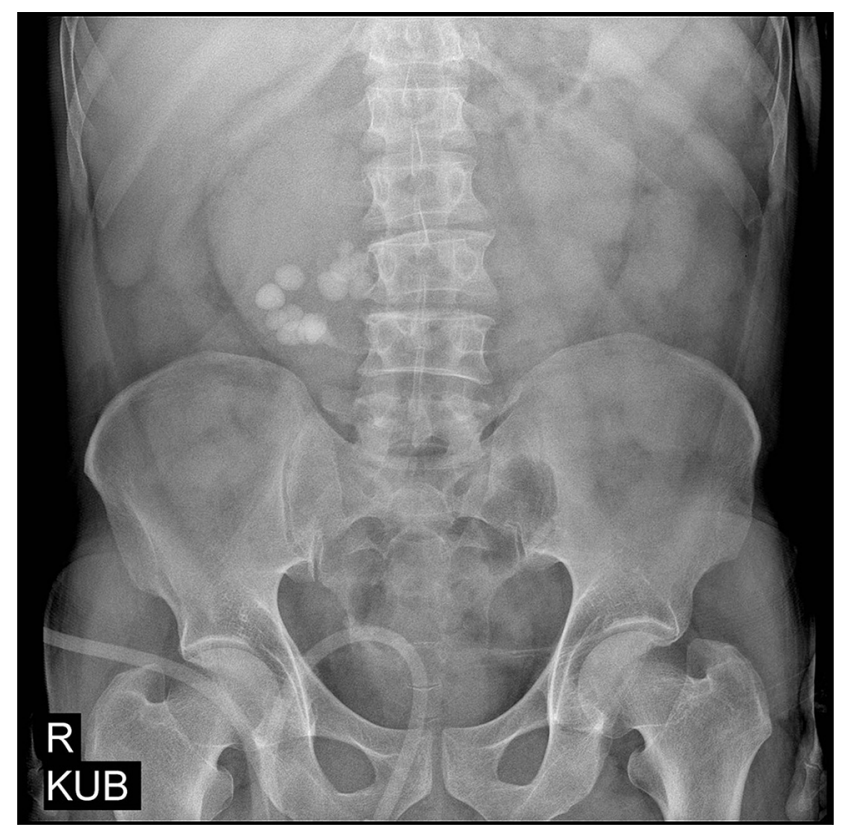

Figure I KUB showing several right renal stones.

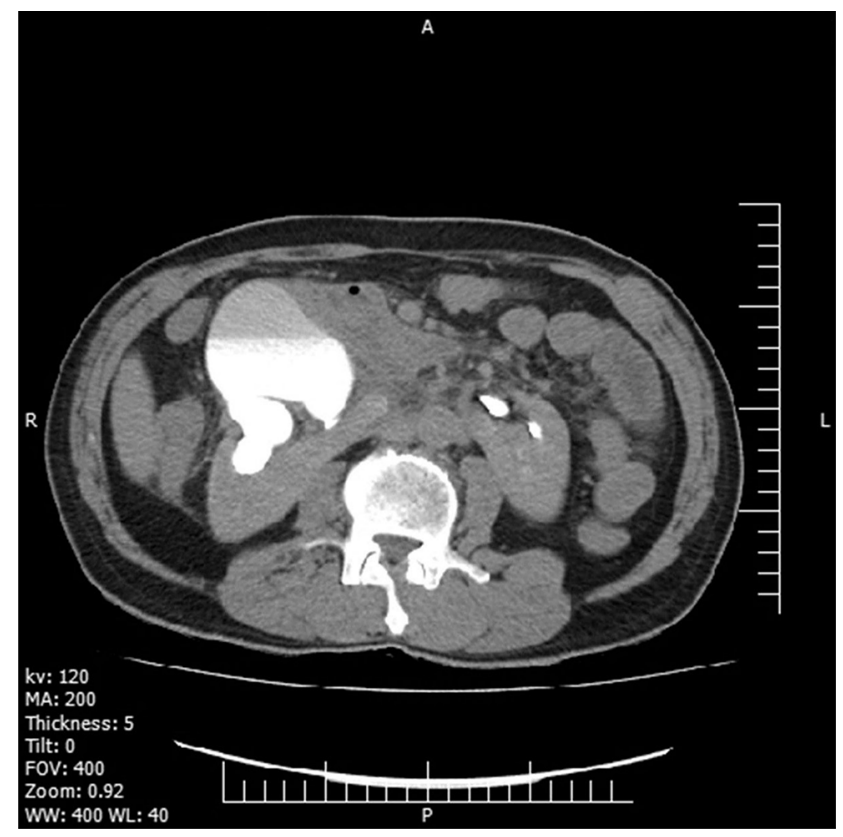

Figure 2 CT-IVU pointing right renal pelvic and lower calyces.

organs injuries. All the procedures and related complications were discussed with the patient and, finally, he was hospitalized for the LAT-PCNL after obtaining the informed consent.

LAT- PCNL was performed under general anesthesia in the semi-flank position. The process began with inserting three laparoscopic ports $11 \mathrm{~mm}$ umbilical, 2 five $\mathrm{mm}$ in subxiphoid, and the right para rectus muscle. The distended pelvis of the right kidney was exposed by mobilizing the overlying duodenum and was completely accessible by anchoring to the anterior abdominal wall using 2-0 nylon suture. Afterward, 10 French (Fr) laparoscope exit the abdomen and Nephroscope was introduced via a $10-\mathrm{mm}$ umbilical port. The Nephroscope was entered through an incision on the anterior surface of distended renal pelvic under the guidance of a smaller size laparoscope entered the $5 \mathrm{Fr}$ right pararectus port (Figure 3). Fluoroscopy was not used throughout the surgery. The abdomen was desufflated, keeping all laparoscopic ports in place. The stones were fragmented using pneumatic lithoclast and the fragmented stones were removed with forceps. At the end of the procedure, a 6 Fr JJ stent was inserted antegrade. The abdomen was reinsufflated and the laparoscope was reinserted to check hemostasis and suturing pyelotomy. A suction drain was placed through a 5-mm port in the surgical field and was removed at discharge day. The surgery lasted about 100 mins. We did not observe any perioperative complication. Postoperative control abdominal x-ray revealed only a middle ureteral stone that was extracted while removing 


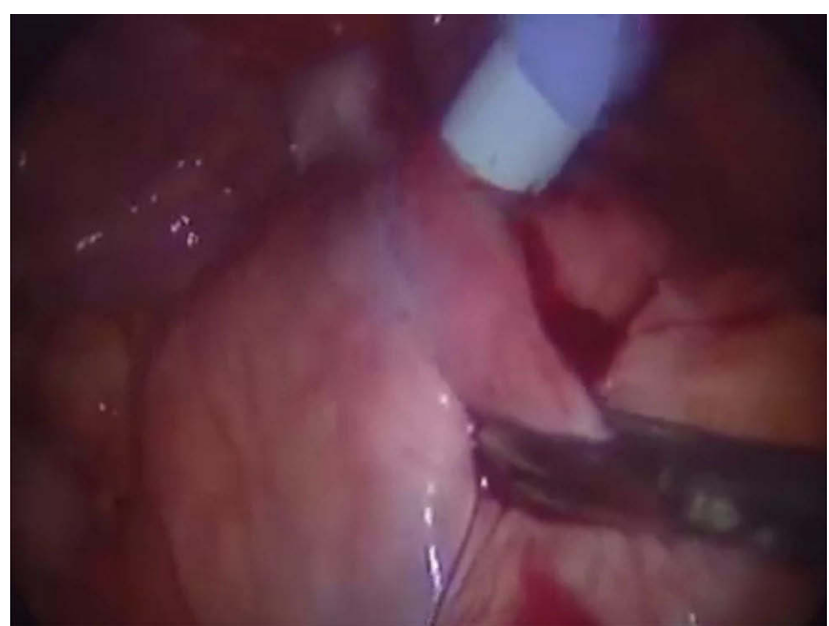

Figure 3 Placement an amplatz sheath over facial dilator into renal pelvis under laparoscopic guidance.

the Double-J stent a month later. The patient was discharged on the third postoperative day with normal laboratory values. At 3-month follow-up, the patient was stone-free with normal renal function and renal ultrasonography.

The patients signed an informed consent containing the aspects of the surgical procedure, the modalities of assisted reproduction, and agreement to the publication of this case report and images.

The study was approved according to North Khorasan University of Medical Sciences (NKUM) and approval ID was IR.MKUMS.REC.1398.128.

\section{Discussion}

Nephrolithiasis is one of the most common complications in horseshoe kidneys. The management of calculi in a horseshoe kidney is a treatment challenge. Different modalities could be applied in the treatment of renal stones in these patients including PCNL, ESWL, flexible ureterorenoscopy, laparoscopy, and even open surgery. ${ }^{3}$ Although conventional PCNL has become leading modality in treating renal stones in HSK with renal stones, the aberrant location, vascular anomalies, and proximity to the peritoneal cavity, sigmoid colon, and iliac vessels all contribute to the difficulty in a standard fluoroscopy-guided percutaneous approach. ${ }^{4}$

In a meta-analysis study conducted by wang et al (2013), the authors assessed the effectiveness and safety of laparoscopic pyelolithotomy versus PCNL as surgical management for solitary renal pelvic calculi larger than $2 \mathrm{~cm}$. They suggested that laparoscopic pyelolithotomy and PCNL are effective and safe for large renal pelvic calculi but laparoscopic pyelolithotomy seems to be more advantageous. ${ }^{5}$ In another study, Eshghi et al reported the successful implementation of laparoscopy-assisted PCNL for the treatment of pelvic kidney stones for the first time. ${ }^{6}$

Santos et al applying a laparoscopic guided percutaneous transperitoneal approach measured a renal pelvic stone of $1.5 \mathrm{~cm},{ }^{7}$ Mousavi-Bahar et al used this technique for 3 cases of ectopic kidney who treated successfully. ${ }^{8}$

Moreover, using the LAT-PCNL for horseshoe kidney calculi, they reported less than renal pelvic calculi. Jiang et al described Retroperitoneoscopy-assisted PCNL in 5 patients with renal stones in a complexity horseshoe kidney. They introduced this technique as a safe, feasible, and helpful procedure in establishing the nephrostomy tract with renal stones in complexity HSK, especially for the HSK that was tightly wrapped by the surrounding organs, such as retrorenal colon. ${ }^{9}$

Maheshwari et al (2004) reported the first laparoscopic management of stone disease in a patient with a horseshoe kidney. They reported the same experience in a patient with horseshoe kidney who achieved a stone-free status in a single-stage operation. They performed PCNL under laparoscopic and fluoroscopic guidance for a direct approach to the Calix containing the stone without extensive dissection or intracorporeal suturing. They used fluoroscope and additional tract, dilators, and Amplatz sheath. ${ }^{10}$

We have the experience of 8 PCNLs and 2 transperitoneal laparoscopic pyelolithotomy in HSKs since 2008. Finally, we propose that laparoscopy-assisted transperitoneal PCNL without fluoroscopy could be a reliable and useful short-duration surgery when the treatment approach is critical due to possible complications. Future studies should prospectively address the outcomes of our approach and patients who benefit from this procedure.

\section{Conclusion}

Laparoscopy-assisted transperitoneal PCNL seems to be a safe and minimally invasive technique that can be used as an alternative approach in the management of renal stones in special cases of horseshoe kidneys.

\section{Disclosure}

The authors report no conflicts of interest in this work.

\section{References}

1. Nishi M, Iwamura M, Kurosaka S, Fujita T, Matsumoto K, Yoshida K Laparoscopic Anderson-Hynes pyeloplasty without symphysiotomy for hydronephrosis with horseshoe kidney. Asian J Endosc Surg. 2013;6(3):192-196. doi:10.1111/ases.2013.6.issue-3 
2. Yohannes P, Smith AD. The endourological management of complications associated with horseshoe kidney. J Urol. 2002;168(1):5-8. doi:10.1016/S0022-5347(05)64819-7

3. Etemadian M, Maghsoudi R, Abdollahpour V, Amjadi M. Percutaneous nephrolithotomy in horseshoe kidney: our 5-year experience. Urol J. 2013;10(2):856-860.

4. Ganpule AP, Prashant J, Desai MR. Laparoscopic and robot-assisted surgery in the management of urinary lithiasis. Arab J Urol. 2012;10 (1):32-39. doi:10.1016/j.aju.2011.12.003

5. Wang X, Li S, Liu T, Guo Y, Yang Z. Laparoscopic pyelolithotomy compared to percutaneous nephrolithotomy as surgical management for large renal pelvic calculi: a meta-analysis. J Urol. 2013;190 (3):888-893. doi:10.1016/j.juro.2013.02.092

6. Eshghi AM, Roth JS, Smith AD. Percutaneous transperitoneal approach to a pelvic kidney for endourological removal of staghorn calculus. J Urol. 1985;134(3):525-527. doi:10.1016/S0022-5347(17) 47274-0
7. Santos ARD, Rocha Filho DC, Tajra LC. Management of lithiasis in pelvic kidney through laparoscopy-guided percutaneous transperitoneal nephrolithotripsy. Int Braz J Urol. 2004;30(1):32-33; discussion 34. doi:10.1590/S1677-55382004000100007

8. Mousavi-Bahar SH, Amir-Zargar MA, Gholamrezaie HR. Laparoscopic assisted percutaneous nephrolithotomy in ectopic pelvic kidneys. Int $J$ Urol. 2008;15(3):276-278. doi:10.1111/ iju.2008.15.issue-3

9. Jiang K, Tang K, Xu H, Chen H, Chen Z. Retroperitoneoscopy technique-assisted percutaneous nephrolithotomy for complexity horseshoe kidney with renal stones. Urol Int. 2016;97(3):285-291. doi: $10.1159 / 000446120$

10. Maheshwari PN, Bhandarkar DS, Shah RS, Andankar MG, Saple AL. Laparoscopy-assisted transperitoneal percutaneous nephrolithotomy for recurrent calculus in isthmic calix of horseshoe kidney. J Endourol. 2004;18(9):858-861. doi:10.1089/end. 2004.18.858

\section{Publish your work in this journal}

Research and Reports in Urology is an international, peer-reviewed, open access journal publishing original research, reports, editorials, reviews and commentaries on all aspects of adult and pediatric urology in the clinic and laboratory including the following topics: Pathology, pathophysiology of urological disease; Investigation and treatment of urological disease; Pharmacology of drugs used for the treatment of urological disease. The manuscript management system is completely online and includes a very quick and fair peer-review system, which is all easy to use. Visit http://www.dovepress.com/ testimonials.php to read real quotes from published authors. 